\title{
ANÁLISE DE TRILHA NO ESTUDO DOS EFEITOS DE ATRIBUTOS FÍSICOS E MATÉRIA ORGÂNICA SOBRE A COMPRESSIBILIDADE E RESISTÊNCIA À PENETRAÇÃO DE UM ARGISSOLO CULTIVADO COM CANA-DE-AÇÚCAR ${ }^{(1)}$
}

\author{
Edson Patto Pacheco ${ }^{(2)}$ \& José Ramon Barros Cantalice ${ }^{(3)}$
}

\begin{abstract}
RESUMO
Uma das formas de avaliar a condição estrutural do solo é por meio da pressão de pré-compactação e da resistência do solo à penetração. Considerando que o conhecimento da associação entre duas variáveis é de grande importância na ciência do solo, o objetivo deste trabalho foi estudar o desdobramento dos efeitos diretos e indiretos, por meio da análise de correlação de trilha ou "path coefficient analysis", de atributos físicos e teor de matéria orgânica sobre a pressão de précompactação média $\left(\sigma_{\mathrm{p}} \mathrm{m}\right)$ e resistência à penetração média em laboratório (RPLm) de um Argissolo Amarelo Distrocoeso cultivado com cana-de-açúcar nos Tabuleiros Costeiros do Estado de Alagoas. $O$ trabalho foi desenvolvido na Fazenda Progresso, de propriedade da Usina Coruripe (AL), onde foram selecionadas cinco áreas cultivadas com cana-de-açúcar com diferentes tempos de uso e dois tipos de cultivo: 4 anos com cultivo de inverno ( 4 anos I); 14 anos com cultivo de inverno (14 anos I); 14 anos com cultivo de verão (14 anos V); 30 anos com cultivo de inverno (30 anos I); 30 anos com cultivo de verão (30 anos V); e uma área de mata (mata), tipo floresta subperenifólia, que foi considerada como testemunha-padrão. As avaliações foram realizadas nas profundidades de 0 a $0,20,0,20$ a 0,40 e 0,40 a $0,60 \mathrm{~m}$, correspondentes aos horizontes A (mata) ou Ap (áreas cultivadas), AB e Bt, respectivamente, para as quais foram realizadas análises de correlação de "trilha" ("path coefficient analysis"), para determinação do grau de associação entre os atributos físicos e o teor de matéria orgânica do solo com a $\sigma_{\mathrm{p}} \mathrm{m}$ e a RPLm (variáveis dependentes). Nas três profundidades, o grau de compactação e a densidade do solo foram os atributos
\end{abstract}

\footnotetext{
(1) Parte da Tese de Doutorado do primeiro autor. Programa de Pós-graduação em Ciência do Solo da Universidade Federal Rural de Pernambuco - UFRPE. Recebido para publicação em março de 2010 e aprovado em dezembro de 2010.

${ }^{(2)}$ Pesquisador da Embrapa Tabuleiros Costeiros. Avenida Beira Mar 3250, Jardins, Caixa Postal 44, CEP 49.025-040 Aracaju (SE). E-mail: patto@cpatc.embrapa.br

(3) Professor do Programa de Pós-Graduação em Ciência do Solo, Universidade Federal Rural de Pernambuco - UFRPE. Departamento de Agronomia. R. Dom Manoel de Medeiros s/n, Dois Irmãos, CEP 52171-900 Recife (PE). E-mail: cantalic@terra.com.br
} 
que mostraram maior relação causa e efeito com a pressão de pré-compactação e resistência à penetração do Argissolo estudado. Nos horizontes A (mata) ou Ap (áreas cultivadas), o teor de matéria orgânica e a estabilidade de agregados em água apresentaram efeito direto sobre as variações da pressão de pré-compactação e resistência à penetração; a mata apresentou maior teor de matéria orgânica, maior porcentagem de agregados estáveis e maior compressibilidade, em relação às áreas cultivadas.

Termos de indexação: Tabuleiros Costeiros, física do solo, pressão de précompactação, correlação linear.

\title{
SUMMARY: PATH COEFFICIENT ANALYSIS OF SOIL PHYSICAL PROPERTIES AND ORGANIC MATTER EFFECTS ON COMPRESSIBILITY AND PENETRATION RESISTANCE OF AN ULTISOL UNDER SUGARCANE
}

\begin{abstract}
One of the ways to evaluate the soil structural status is by means of the pre-compaction pressure and soil penetration resistance. In view of the importance of associations between two variables in soil science, the objective of this study was the decomposition of the direct and indirect effects ("path coefficient analysis") of soil physical properties and organic matter on pre-compaction pressure $(\sigma p)$ and laboratory-determined soil penetration resistance (LPS) of a cohesive Yellow Ultisol under sugarcane in the coastal tablelands of the state of Alagoas. The study was conducted on the Fazenda Progresso, of the Usina Coruripe, where five sugarcane areas were selected representing a range of cropping periods and systems: four years under winter cropping (4 year W), 14 years under winter cropping (14 year W), 14 years under summer cropping (14 year $S)$, 30 years under winter cropping (30 year $W$ ), and 30 years under summer cropping (30 year $S$ ), A native subperennial forest fragment (Forest) was also included in this study as reference area. Path coefficient analyses were performed for the layers $0-0.20$, $0.20-0.40$, and $0.40-0.60 \mathrm{~m}$ to assess the degree of association of soil physical properties and soil organic matter with $\sigma p$ and LPS. In all layers, the cause/effect relationship of the compaction degree and soil bulk density with $\sigma p$ and LPS of the studied Ultisol was highest. In the A or Ap horizons, soil organic matter and water aggregate stability had a direct effect on the shifts in $\sigma p$ and LPS; organic matter, percentage of water stable aggregates and compressibility were higher under native forest than in the agricultural areas.
\end{abstract}

Index terms: Coastal tablelands, soil physics, pre-compaction pressure, linear correlation.

\section{INTRODUÇÃO}

Uma das formas de estimar a compactação do solo é por meio da pressão de pré-compactação $\left(\sigma_{p}\right)$, que é determinada pela curva de compressão obtida com ensaio de adensamento uniaxial (ABNT, 1990; Dias Junior, 2000). Essa variável determina a maior pressão que o solo já sofreu no passado e representa a capacidade de suporte de carga, ou seja, é a pressão máxima que o solo suporta antes que ocorra compactação adicional (Dias Junior \& Pierce, 1996). A $\sigma_{\mathrm{p}}$ divide a curva de compressão em duas regiões distintas: curva de compressão secundária; e reta de compressão virgem (Lebert \& Horn, 1991; Dias Junior \& Pierce, 1996). A curva de compressão secundária representa os níveis de pressões sofridas pelo solo no passado, enquanto a reta de compressão virgem representa as primeiras pressões aplicadas ao solo que poderão resultar em compactação adicional. É na região da curva de compressão secundária que o solo deve ser cultivado ou trafegado, sem que ocorram compactações adicionais (Dias Junior \& Pierce, 1996).

A $\sigma_{\mathrm{p}}$ está relacionada com a resistência do solo à penetração das raízes; valores elevados de pressão de pré-compactação apresentam maior probabilidade de reduzir o crescimento das raízes (Römkens \& Miller, 1971). Considerando que a $\sigma_{\mathrm{p}}$ e a resistência do solo à penetração variam em função da umidade e que existe relação significativa, positiva e linear entre as duas variáveis, pode-se afirmar que uma pode ser estimada a partir da outra, por meio de modelos matemáticos (Lima \& Silva, 2006).

Segundo Gupta \& Allmaras (1987), a facilidade com que o solo não saturado decresce de volume, quando submetido a pressões externas, é chamada de compressibilidade, a qual depende de fatores internos e externos (Lebert \& Horn, 1991). Estes autores 
consideram que fatores externos são caracterizados pelo tipo, pela intensidade e pela frequência da carga aplicada. Os internos correspondem ao histórico de tensão, umidade do solo, textura do solo, estrutura do solo, densidade inicial do solo e teor de $\mathrm{C}$ de compostos orgânicos do solo; para uma mesma condição, é a umidade que governa a quantidade de deformação que poderá ocorrer no solo (Bigner \& Wells, 1992; Dias Junior, 1994; Etana et al., 1997; Braida et al., 2006).

O conhecimento da associação entre duas variáveis é de grande importância nos trabalhos de ciência do solo. O coeficiente de correlação linear simples indica que há relação linear entre duas variáveis, e a mudança de uma implica mudança constante no valor médio de outra variável. No entanto, uma vez que na ciência do solo os fatores ambientais relacionam-se de maneira interativa e complexa, o estudo da correlação simples torna-se insuficiente (Gomes, 1996). Portanto, a análise de trilha ou "path coeficient analysis" fornece valores, chamados de coeficiente de trilha, que medem a influência direta de uma variável sobre a outra, independentemente das demais, no contexto de causa e efeito. Esse tipo de análise também permite desdobrar coeficientes de correlação simples em seus efeitos diretos e indiretos (Cruz et al., 2004).

O objetivo deste trabalho foi estudar o desdobramento dos efeitos diretos e indiretos, por meio da análise de correlação de trilha ou "path coefficient analysis", de atributos físicos e do teor de matéria orgânica sobre a pressão de pré-compactação média e resistência à penetração em laboratório média de um Argissolo Amarelo Distrocoeso cultivado com cana-deaçúcar nos Tabuleiros Costeiros do Estado de Alagoas.

\section{MATERIAL E MÉTODOS}

O trabalho foi desenvolvido em áreas localizadas entre os paralelos $10^{\circ} 01$ ' 59 " e $10^{\circ} 02$ ' 58 " S e os meridianos $36^{\circ} 10$ ' 22 " e $36^{\circ} 12$ ' 35 ” W, da Fazenda Progresso, de propriedade da Usina Coruripe, localizada no município de Coruripe-AL. O clima do local é tropical chuvoso com verão seco, segundo a classificação de Köppen. A pluviosidade média anual é de aproximadamente $1.400 \mathrm{~mm}$, e a temperatura média, de $24,4{ }^{\circ} \mathrm{C}$. O solo avaliado foi classificado como Argissolo Amarelo distrocoeso, relevo plano, textura média (leve)/argilosa (Embrapa, 2006), formado do sedimento Grupo Barreiras, característico da unidade geomorfológica Tabuleiros Costeiros (Jacomini et al., 1975).

Foram selecionadas cinco áreas cultivadas com cana-de-açúcar, representando diferentes tempos de uso e dois tipos de cultivo: 14 anos com cultivo de inverno (4 anos I); 14 anos com cultivo de inverno (14 anos I); 14 anos com cultivo de verão (14 anos V); 30 anos com cultivo de inverno (30 anos I); 30 anos com cultivo de verão (30 anos V); e uma área de mata
(Mata), tipo floresta subperenifólia, que foi considerada testemunha-padrão, totalizando seis tratamentos.

Conforme informações obtidas na Usina Coruripe, os sistemas de plantio de inverno e verão para renovação dos canaviais apresentam as características descritas a seguir:

Plantio de inverno: durante o mês de abril é realizada semeadura de Crotalaria spectabilis, a lanço com avião, seguida de subsolagem $(0,60 \mathrm{~m}$ de profundidade). Após o desenvolvimento vegetativo da leguminosa, é realizada a abertura de sulcos, o plantio da cana e a aplicação de herbicida (paraquat + metribuzin), durante os meses de julho/agosto (inverno chuvoso).

Plantio de verão irrigado: durante todo o período da colheita, que ocorre de setembro a março (verão seco), é realizado o preparo do solo com uma gradagem média (discos 26") para posterior subsolagem $(0,60 \mathrm{~m}$ de profundidade) e abertura dos sulcos de plantio, onde é aplicado o composto orgânico na dose de 30 t ha ${ }^{-1}$. Após o plantio, é aplicado herbicida pré-emergente (metribuzin).

Nos dois sistemas, após cada colheita da cana queimada, é realizada uma tríplice operação de escarificação/adubação/cultivo, que ocorre durante os meses de setembro a março. Durante o ciclo vegetativo da cana são realizados tratos fitossanitários por meio de pulverizadores costais, mecanizados ou por aviação agrícola.

Para realização das análises físicas e da matéria orgânica, foram retiradas amostras de solo em quatro repetições para cada área, nas profundidades de 0 a 0,20, 0,20 a 0,40 e 0,40 a 0,60 m, correspondendo aos horizontes A (mata) ou Ap (áreas cultivadas), AB e $\mathrm{Bt}$, respectivamente.

A composição textural do solo foi determinada pelo método do densímetro de Boyoucos (Embrapa, 1997), cujas médias dos teores de areia, silte e argila dos seis tratamentos, nas três profundidades estudadas, são apresentadas no quadro 1.

Para determinação da pressão de pré-compactação $\left(\sigma_{\mathrm{p}}\right)$, resistência do solo à penetração em laboratório (RPL), densidade do solo (Ds), volume total de poros (VTP), porcentagem de microporos (MICRO) e de macroporos (MACRO), foram utilizadas amostras indeformadas extraídas do solo com anéis volumétricos de PVC com 0,052 $\mathrm{m}$ de diâmetro e 0,020 $\mathrm{m}$ de altura, por meio de trado de Uhland, conforme descrito por Pacheco (2010).

As amostras indeformadas foram saturadas por $24 \mathrm{~h}$, para posterior estabilização da umidade em oito sucções de potenciais matriciais $(-1,-4,-6,-10,-33$, 100, -500; e -1.500 kPa), utilizando mesa de tensão e câmara de Richards (Embrapa, 1997). Estabilizado o teor de água, as amostras foram submetidas ao ensaio de RPL, utilizando um penetrógrafo de bancada com 
Quadro 1. Caracterização granulométrica dos horizontes A (mata) ou Ap (áreas cultivadas), AB e Bt das seis áreas estudadas

\begin{tabular}{|c|c|c|c|c|c|c|c|c|c|}
\hline \multirow{2}{*}{ Tratamento } & \multicolumn{3}{|c|}{ Horizonte A ou Ap } & \multicolumn{3}{|c|}{ Horizonte AB } & \multicolumn{3}{|c|}{ Horizonte Bt } \\
\hline & Areia & Silte & Argila & Areia & Silte & Argila & Areia & Sil te & Argila \\
\hline & \multicolumn{9}{|c|}{$\mathrm{g} \mathrm{kg}^{-1}$} \\
\hline Mata & 868 & 57 & 76 & 793 & 56 & 151 & 632 & 78 & 291 \\
\hline 4 anos I & 867 & 46 & 88 & 701 & 65 & 234 & 596 & 102 & 326 \\
\hline 14 anos I & 866 & 49 & 86 & 751 & 66 & 184 & 592 & 102 & 306 \\
\hline 14 anos $\mathrm{V}$ & 873 & 42 & 86 & 768 & 63 & 169 & 606 & 97 & 297 \\
\hline 30 anos I & 885 & 39 & 75 & 747 & 81 & 172 & 608 & 127 & 265 \\
\hline 30 anos V & 868 & 42 & 91 & 724 & 70 & 207 & 593 & 119 & 288 \\
\hline
\end{tabular}

cone de 4 mm de diâmetro, ângulo de $45^{\circ}$ e velocidade de penetração de $10 \mathrm{~mm} / \mathrm{min}$ (Pacheco, 2010).

Após o ensaio de RPL, as amostras foram pesadas (peso do solo úmido) e submetidas ao ensaio de compressão uniaxial, utilizando uma prensa manual, onde foram aplicadas cargas verticais correspondentes a tensões de 12,$5 ; 25 ; 50 ; 100 ; 200 ; 400 ; 800$; e $1.600 \mathrm{kPa}$, segundo norma de Ensaio de Adensamento Unidimensional - MB 3336 da ABNT (1990). A razão de carga foi igual a 2, e os estádios de carregamentos, de 30 s. Para cada carga aplicada, foi medida a deformação vertical da amostra, por meio de micrômetro com precisão de $0,01 \mathrm{~mm}$, conforme descrito por Pacheco (2010). Ao final do ensaio de compressão, as amostras foram secas em estufa a $105^{\circ} \mathrm{C}$ por $24 \mathrm{~h}$, para determinação da massa do solo seco.

A entrada dos dados de densidade de partícula (método do picnômetro - Embrapa (1997), da massa do solo úmido, da massa do solo seco, dimensões do anel de PVC e valor da deformação vertical (para cada carga aplicada) foi realizada no programa "Compress" (Reinert et al., 2003), para determinação da pressão de pré-compactação $\left(\sigma_{p}\right)$, por meio da curva de compressão. O método utilizado na determinação da $\sigma_{\mathrm{p}}$ foi o Pacheco e Silva - 03, descrito na norma MB 3336 da ABNT (1990). Por meio do programa "Compress" também foram determinados a Ds, VTP, microporosidade (água retida na tensão de $6 \mathrm{kPa}$ ) e macroporosidade (VTP - microporosidade).

A análise de agregados estáveis em água foi feita com adaptações do método de peneiramento úmido, em aparelho de oscilação vertical (Kemper \& Rosenau, 1986). Os valores de agregação (AGR), de agregados estáveis em água (AEA) e diâmetro médio ponderado de agregados estáveis em água (DMP) foram determinados conforme adaptações do método da Embrapa (1997), descrito por Pacheco (2010).

O grau de compactação foi calculado por meio da relação entre a densidade do solo (Ds) e a densidade máxima obtida pela curva de compactação do ensaio de Proctor, conforme descrito por Caputo (1980): $C G$ $=(D s / D M)^{*} 100$, em que: $G C=$ grau de compactação (\%); $D s=$ densidade do solo $\left(\mathrm{kg} \mathrm{dm}^{-3}\right)$; e $D M=$ densidade máxima do solo $\left(\mathrm{kg} \mathrm{dm}^{-3}\right)$. Para obtenção das curvas de compactação foi utilizado um "Proctor" com dimensões reduzidas em relação ao aparelho considerado na norma NBR 7182 (ABNT, 1986), que utiliza $1 \mathrm{dm}^{3}$ de solo para cada ensaio, o que implica grande volume de solo a ser transportado, quando o número de testes é grande, com amostras não reaproveitadas para diferentes umidades. Portanto, foi construído um "Proctor" que comporta, em um cilindro de ferro, amostras com $113,70 \mathrm{~cm}^{3}(0,0538 \mathrm{~m}$ de diâmetro e $0,0500 \mathrm{~m}$ de altura). As amostras receberam 12 golpes de um martelo cilíndrico com peso de 1,8 kg, com 0,305 m de altura de queda, para obtenção da energia de compactação similar à do ensaio de Proctor normal, conforme descrito por Pacheco (2010).

O teor de matéria orgânica total (MOT) foi determinado por oxidação via húmida, com titulação do excesso de bicromato de $\mathrm{K}$ pelo sulfato ferroso amoniacal, conforme descrito por Embrapa (1999). O fracionamento físico para determinação da matéria orgânica particulada (MOP) foi realizado segundo Cambardella \& Elliot (1992). Para isso, erlenmeyers de $250 \mathrm{~mL}$, contendo amostras de $20 \mathrm{~g}$ de terra fina seca ao ar e $70 \mathrm{~mL}$ de solução de hexametafosfato de sódio $\left(5 \mathrm{~g} \mathrm{~L}^{-1}\right)$, foram agitados durante $15 \mathrm{~h}$ em agitador horizontal. A seguir, a suspensão foi lavada em peneira de $0,053 \mathrm{~mm}$, com auxílio de jato d'água. $\mathrm{O}$ material retido na peneira, que consiste em areia $\mathrm{e}$ matéria orgânica particulada (>0,053 mm), foi seco em estufa a $50{ }^{\circ} \mathrm{C}$. Após ter sido quantificada sua massa, em balança com precisão de 0,01 g, o material foi moído em gral de porcelana e analisado em relação ao teor de matéria orgânica (MO), por oxidação, com titulação do excesso de bicromato de potássio pelo sulfato ferroso amoniacal. O teor de matéria orgânica associada aos minerais ou matéria orgânica complexada (MOC) foi calculado pela diferença entre os teores de MOT e MOP. 
Para determinação do grau de associação, entre os atributos físicos e do teor de matéria orgânica do solo com a pressão de pré-compactação média $\left(\sigma_{\mathrm{p}} \mathrm{m}\right)$ e resistência à penetração média em laboratório (RPLm), foram realizadas análises de correlação de "trilha" (análise de trilha ou "path coefficient analysis"). Esse tipo de análise de correlação fornece quantidades, chamadas coeficientes de trilha, que medem a influência direta de uma variável sobre outra, independentemente das demais, no contexto das relações de causa e efeito. Para essa análise, foi utilizado o programa SAEG 9.1, desenvolvido pela Universidade Federal de Viçosa. Foram consideradas como variáveis dependentes a pressão de précompactação $\left(\sigma_{p}\right)$ e a resistência do solo à penetração (RPL). Como variáveis explicativas, foram considerados cinco grupos de atributos do solo: (1) Textura (areia, silte e argila); (2) estabilidade de agregados em água por meio da: agregação (AGR), agregados estáveis em água (AEA) e diâmetro médio de agregados estáveis em água (DMP); (3) porosidade (densidade do solo (Ds), volume total de poros (VTP), volume de microporos (VMicro) e volume de macroporos (VMacro)); (4) compactabilidade (grau de compactação (GC) e densidade máxima (DM)); e (5) teor de matéria orgânica (matéria orgânica total (MOT), matéria orgânica particulada (MOP), matéria orgânica complexada (MOC), proporção de MOP (MOP) e percentagem de MOC (MOC)).

Para cada análise de trilha foi composto um conjunto de variáveis; foi selecionada, sempre que possível, pelo menos uma variável explicativa de cada grupo de atributo. Para que a análise de trilha apresente consistência, o conjunto de variáveis deve apresentar colinearidade fraca. Assim, as seleções foram realizadas com base em análises de multicolinearidade realizadas por meio do programa GENES versão 2007.

As seguintes propriedades têm sido associadas aos efeitos da análise de trilha: (a) sendo um coeficiente de regressão, ele tem direção, podendo ser positivo ou negativo e maior ou menor do que a unidade; (b) sendo um coeficiente de regressão padronizado, ele pode ser utilizado para comparar efeito de caracteres mensuráveis em diferentes escalas; e (c) não tendo unidade física, ele se assemelha a um coeficiente de correlação (Cruz et al., 2004). Essa observação permite que os coeficientes de trilha (efeitos diretos) possam ser comparados entre si e com o efeito da variável residual, que é utilizado como referencial para a importância do efeito direto das variáveis explicativas sobre a variável principal ou dependente. Quando o coeficiente de trilha (efeito direto) de uma variável explicativa for, em módulo, menor do que o coeficiente da variável residual $(\rho \varepsilon)$, mas o coeficiente de correlação (efeito total) for maior, em módulo, do que o efeito da variável residual, significa que essa variável explicativa tem efeito sobre a variável principal apenas indiretamente, sendo sua importância só em conjunto. Se o coeficiente de trilha for, em módulo, maior do que o coeficiente da variável residual ( $\rho \varepsilon)$, indica que existe efeito direto da variável explicativa sobre a principal (Cruz et al., 2004). Variáveis que apresentam coeficiente de trilha com mesmo sinal e magnitude do coeficiente de correlação são determinantes do comportamento da variável dependente. No entanto, quando uma variável explicativa apresenta coeficiente de trilha com sinal contrário ao do coeficiente de correlação, significa que essa variável não apresenta relação de causa e efeito direto com a variável principal, devendo ser considerados somente seus efeitos indiretos em conjunto com outras variáveis (Gomes, 1996).

\section{RESULTADOS E DISCUSSÃO}

Os coeficientes de correlação, de trilha e dos efeitos indiretos das variáveis de atributos físicos e teor de matéria orgânica do Argissolo estudado sobre a $\sigma_{\mathrm{p}} \mathrm{m}$, na profundidade de 0 a $0,20 \mathrm{~m}$, são apresentados no quadro 2.

Quadro 2. Desdobramento das correlações lineares em efeitos diretos e indiretos de atributos físicos e teor de matéria orgânica sobre a $\sigma_{\mathrm{p}} \mathrm{m}$ para a profundidade de 0 a $0,20 \mathrm{~m}$

\begin{tabular}{lrrrrrrr}
\hline Variável & S+A & MOT & PMOP & AGR & Vmicro & GC & Total \\
\hline S+A & $\mathbf{- 0 , 2 7 0}$ & 0,038 & 0,000 & 0,108 & $-0,129$ & 0,298 & 0,045 \\
MOT & 0,050 & $\mathbf{- 0 , 1 9 7}$ & 0,051 & $-0,289$ & 0,091 & $-0,390$ & $-0,684^{* *}$ \\
PMOP & $-0,003$ & $-0,133$ & $\mathbf{0 , 0 7 5}$ & $-0,246$ & 0,038 & $-0,400$ & $-0,669^{* *}$ \\
AGR & 0,087 & $-0,170$ & 0,055 & $-\mathbf{0 , 3 3 4}$ & 0,102 & $-0,451$ & $-0,711^{* *}$ \\
Vmicro & 0,151 & $-0,078$ & 0,012 & $-0,147$ & $\mathbf{0 , 2 3 1}$ & $-0,181$ & $-0,012$ \\
GC & $-0,113$ & 0,107 & $-0,042$ & 0,211 & $-0,058$ & $\mathbf{0 , 7 1 3}$ & $0,818^{* *}$ \\
Coeficiente de Determinação $\left(R^{2}\right)$ & & & & & 0,890 \\
Efeito da Variável Residual $(\rho \varepsilon)$ & & & & & & 0,331 \\
Colinearidade & & & & & & Fraca
\end{tabular}

* correlação de Pearson significativa a $5 \%$, para o efeito total; ** correlação de Pearson significativa a 1 \%, para o efeito total; Leitura dos efeitos diretos (coeficiente de trilha) sobre a variável resposta na diagonal, em negrito, e dos efeitos indiretos na horizontal; - S+A: silte mais argila; MOT: teor de matéria orgânica total; PMOP: porcentagem de matéria orgânica particulada; AGR: porcentagem de agregação em água; Vmicro: volume de microporos; e GC: grau de compactação. 
O conjunto das variáveis selecionadas explicou $89,0 \%\left(\mathrm{R}^{2}\right)$ das variações na pressão de précompactação e apresentou colinearidade fraca (Quadro 2). As variáveis que apresentaram coeficiente de correlação (efeito total) significativo a $1 \%$ com a $\sigma_{\mathrm{p}} \mathrm{m}$ podem ser classificadas na seguinte ordem decrescente: GC> AGR> MOT> PMOP. O GC teve correlação positiva, e as demais variáveis, correlação negativa com a variável $\sigma_{\mathrm{p}} \mathrm{m}$. Esse resultado indica que o aumento do GC aumentou a capacidade de suporte de carga do solo $\left(\sigma_{\mathrm{p}}\right)$ e que o aumento da estabilidade de agregados e teor de matéria orgânica favoreceu a maior compressibilidade na profundidade de 0 a $0,20 \mathrm{~m}$ do Argissolo Amarelo estudado.

Somente o GC e a AGR apresentaram coeficiente de trilha maior do que o efeito da variável residual e com mesmo sinal do efeito total, indicando a importância da relação causa e efeito dessas duas variáveis com a $\sigma_{\mathrm{p}} \mathrm{m}$ (Quadro 2). A variável GC foi a mais importante no efeito direto sobre a $\sigma_{\mathrm{p}} \mathrm{m}$, pois apresentou o maior coeficiente de trilha com mesmo sinal e magnitude semelhante à do efeito total. A AGR teve coeficiente de trilha com magnitude muito inferior à do efeito total, indicando sua menor importância na relação de causa e efeito direto com a $\sigma_{\mathrm{p}} \mathrm{m}$.

As variáveis AGR, MOT e PMOP apresentaram coeficientes de correlação para efeito indireto, via GC, igual a -0,451, -0,390 e -0,400, respectivamente (Quadro 2). Em módulo, esses coeficientes são maiores do que o efeito residual $(0,331)$, indicando que o teor de matéria orgânica e a estabilidade de agregados favoreceram o menor grau de compactação do solo e, consequentemente, sua maior compressibilidade.

Os efeitos totais, diretos e indiretos das variáveis de atributos físicos e matéria orgânica do Argissolo estudado sobre a RPLm, na profundidade de 0 a $0,20 \mathrm{~m}$, estão apresentados no quadro 3.

O conjunto de variáveis selecionado explicou 90,4 \% $\left(\mathrm{R}^{2}\right)$ das variações na resistência à penetração $\mathrm{e}$ mostrou colinearidade fraca.
As variáveis que superaram o efeito da variável residual, em ordem decrescente para coeficiente de correlação (efeito total), foram GC $>\mathrm{MOT}>\mathrm{AGR}$, apresentando significância a $1 \%$. A grande importância da relação de causa e efeito do GC e da MOT, com a RPL do horizonte superficial do Argissolo, é expressa pelos coeficientes de trilha de mesmo sinal e magnitude semelhante à apresentada pelos coeficientes de correlação; o GC pode ser considerado a variável explicativa mais importante, por apresentar o maior coeficiente de trilha (Quadro 3).

Mesmo apresentando efeito total significativo, a AGR não apresentou relação causa e efeito com a variável dependente, pois seu coeficiente de trilha apresentou sinal contrário ao do coeficiente de correlação. No entanto, seu efeito indireto via GC maior do que o efeito da variável residual confirma a importância da agregação do solo na diminuição do grau de compactação e, consequentemente, na menor resistência do solo à penetração de raízes.

Na profundidade de 0 a $0,20 \mathrm{~m}$, o solo da mata apresentou teor de MOT de 17,80 $\mathrm{g} \mathrm{kg}^{-1}$, que, relativamente, foi bem superior aos teores das áreas cultivadas, os quais variaram de 8,72 a $11,54 \mathrm{~g} \mathrm{~kg}^{-1}$ (Quadro 4). O sistema de colheita de cana queimada, combinado com tratos culturais que movimentam o solo excessivamente nas entrelinhas, pode ter contribuído para a redução do teor de MOT, que é um dos principais fatores responsáveis pela formação de agregados na camada superficial do solo, que apresentou $84,1 \%$ de agregação na mata, contra uma variação de 22,36 a 35,11 \% de agregação nas áreas cultivadas (Quadro 4).

Segundo Horn et al. (1993), a resistência do solo ao cisalhamento tende a aumentar com o desenvolvimento da estrutura. Entretanto, D'Agostini et al. (1992) argumentam que solos recuperados por processos biológicos, em que as condições estruturais se aproximam das naturais, tendem a apresentar condições de elevada compressibilidade (baixa $\sigma_{\mathrm{p}}$ ), uma

Quadro 3. Desdobramento das correlações lineares em efeitos diretos e indiretos de atributos físicos e teor de matéria orgânica sobre a resistência do solo à penetração em laboratório média $\left(\mathrm{RPL}_{\mathrm{m}}\right) \mathrm{para}^{\mathrm{a}}$ profundidade de 0 a $0,20 \mathrm{~m}$

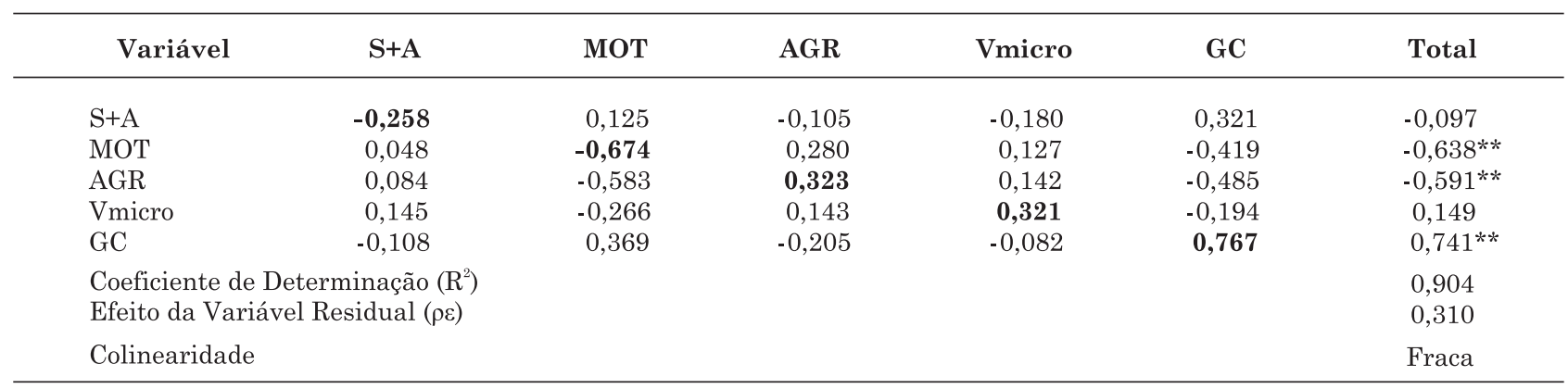

* correlação de Pearson significativa a $5 \%$, para o efeito total; ** correlação de Pearson significativa a 1 \%, para o efeito total; Leitura dos efeitos diretos (coeficiente de trilha) sobre a variável resposta na diagonal, em negrito, e dos efeitos indiretos na horizontal; - S+A: silte mais argila; MOT: teor de matéria orgânica total; AGR: percentagem de agregação em água; Vmicro: volume de microporos; e GC: grau de compactação. 
Quadro 4. Pressão de pré-compactação média, resistência do solo à penetração média em laboratório, atributos físicos e teor de matéria orgânica do Argissolo dos seis tratamentos na profundidade de 0 a 0,20 m

\begin{tabular}{|c|c|c|c|c|c|c|}
\hline \multirow{2}{*}{ Variável } & \multicolumn{6}{|c|}{ Tratamento } \\
\hline & Mata & $4 \operatorname{anos} I$ & 14 anos $I$ & 14 anos $V$ & 30 anos $I$ & 30 anos $V$ \\
\hline$\sigma_{\mathrm{p}} \mathrm{m}(\mathrm{kPa})$ & 19 & 28 & 29 & 36 & 39 & 40 \\
\hline RPLm (kPa) & 186 & 356 & 310 & 414 & 477 & 699 \\
\hline $\mathrm{S}+\mathrm{A}\left(\mathrm{g} \mathrm{kg}^{-1}\right)$ & 132 & 133 & 134 & 127 & 115 & 132 \\
\hline MOT $\left(\mathrm{g} \mathrm{kg}^{-1}\right)$ & 17,80 & 9,39 & 11,54 & 10,84 & 9,97 & 8,72 \\
\hline PMOP (\%) & 22,44 & 15,28 & 15,71 & 16,65 & 15,31 & 11,27 \\
\hline PMOC (\%) & 77,56 & 84,72 & 84,29 & 83,35 & 84,69 & 88,73 \\
\hline AGR (\%) & 84,10 & 34,67 & 35,11 & 31,69 & 22,36 & 31,26 \\
\hline AEA (\%) & 90,88 & 63,80 & 63,09 & 67,31 & 59,49 & 64,64 \\
\hline $\mathrm{DMP}(\mathrm{mm})$ & 2,36 & 0,84 & 0,79 & 0,95 & 0,64 & 0,79 \\
\hline $\operatorname{VTP}\left(\mathrm{m}^{3} \mathrm{~m}^{-3}\right)$ & 0.53 & 0.47 & 0.47 & 0.47 & 0.45 & 0.44 \\
\hline MICRO (\%) & 33.18 & 32.58 & 34.75 & 34.14 & 35.41 & 36.11 \\
\hline MACRO (\%) & 66.82 & 67.42 & 65.25 & 65.86 & 64.59 & 63.89 \\
\hline Ds $\left.(\mathrm{kg} \mathrm{dm})^{-3}\right)$ & 1,21 & 1,38 & 1,37 & 1,39 & 1,44 & 1,46 \\
\hline GC (\%) & 71,44 & 72,60 & 74,07 & 75,12 & 77,09 & 77,15 \\
\hline
\end{tabular}

$\sigma_{\mathrm{p}} \mathrm{m}$ : pressão de pré-compactação média; RPLm: resistência do solo à penetração em laboratório média; S+A: silte mais argila; MOT: matéria orgânica total; PMOP: porcentagem de matéria orgânica particulada; PMOC: porcentagem de matéria orgânica complexada; AGR: porcentagem de agregação em água; AEA: porcentagem de agregados estáveis em água; DMP: diâmetro médio ponderado de agregados estáveis em água; VTP: volume total de poros; MICRO: porcentagem microporos; MACRO: porcentagem macroporos; Ds: densidade do solo; e GC: grau de compactação.

vez que a agregação é acompanhada de aumento da porosidade, em especial da macroporosidade. Um solo que apresenta macroporosidade elevada é mais compressível, porque apresenta menor número de pontos de contato entre partículas sólidas para conferir resistência mecânica à pressão compressiva imposta a ele. Portanto, a matéria orgânica apresenta efeitos contraditórios sobre a resistência do solo à compactação: aumento da força de ligação entre as partículas minerais; e mudança no arranjo das partículas (porosidade) (Horn \& Libert, 1994).

O balanço entre esses dois efeitos, que por sua vez depende da origem e do estado de decomposição da matéria orgânica, irá determinar o seu efeito sobre a resistência do solo. De fato, a matéria orgânica pode aumentar ou reduzir a resistência do solo, dependendo do seu efeito conjunto sobre densidade, parâmetros de cisalhamento, tensão capilar e grau de saturação de água (Zhang et al., 1997). Quando predominam os efeitos de redução da densidade, por aumento da porosidade, a resistência à compressão diminui. Entretanto, a matéria orgânica produz aumento na resistência do solo à compressão, quando predomina o efeito de aumento dos parâmetros de cisalhamento, aumento da tensão capilar com aumento da coesão aparente e redução do efeito da água na redução da fricção entre partículas, redução da densidade por efeito de diluição e aumento da elasticidade (Zhang et al., 1997).

O maior teor de matéria orgânica e a agregação do horizonte superficial do solo da mata também conferiram ao solo desse tratamento maior VTP, menor Ds e menor GC, em relação às áreas cultivadas. A modificação desses atributos, no cultivo com a cana-de-açúcar, teve efeitos diretos e indiretos sobre o comportamento mecânico do Argissolo estudado, conferindo-lhe tendência de aumento da pressão de pré-compactação e resistência à penetração com o tempo de uso (Quadro 4). Ainda, observa-se tendência de maior pressão de pré-compactação e resistência à penetração das áreas sob cultivo de verão, em relação às de inverno, para 14 e 30 anos de uso. Esse fato parece ser relacionado com a maior movimentação do horizonte Ap no sistema de cultivo de verão e no cultivo de leguminosas nas áreas de inverno, que também mostraram tendência de maior teor de MOT e menor Ds.

No quadro 5 são apresentados os resultados da análise de trilha, com desdobramento dos efeitos diretos e indiretos de atributos de física do solo e teor de matéria orgânica sobre a pressão de précompactação do horizonte $\mathrm{AB}$. O conjunto de variáveis selecionado explicou $87,2 \%\left(\mathrm{R}^{2}\right)$ das variações na $\sigma_{\mathrm{p}} \mathrm{m}$ e mostrou colinearidade fraca.

$\mathrm{Na}$ profundidade de 0,20 a 0,40 m, as variáveis DMP, AGR e PMOP apresentaram coeficientes de correlação significativos e negativos com a $\sigma_{p} m$. Essas variáveis foram pouco importantes na relação de causa e efeito com a variável principal, por apresentarem coeficientes de trilha e de efeitos indiretos inferiores ao efeito da variável residual (Quadro 5). No entanto, o DMP e a AGR apresentaram coeficiente de efeito indireto, via Ds, bem próximo do valor do efeito da variável residual, indicando que, mesmo para profundidades abaixo de $0,20 \mathrm{~m}$, a agregação do Argissolo tende a ter importância sobre a relação de causa e efeito com sua qualidade estrutural, ou seja, maior compressibilidade devido à menor compactação.

As variáveis explicativas (silte + argila) $\mathrm{S}+\mathrm{A}$ e Ds mostraram coeficiente de correlação significativo com sinal positivo; a densidade do solo foi a variável mais importante na relação de causa e efeito com a pressão 
de pré-compactação, apresentando coeficiente de trilha superior ao da variável explicativa $\mathrm{S}+\mathrm{A}$ (Quadro 5).

Os efeitos totais das variáveis explicativas sobre a RPLm foram similares aos resultados obtidos para $\sigma_{\mathrm{p}} \mathrm{m}$. O conjunto de variáveis selecionado explicou $84,7 \%\left(\mathrm{R}^{2}\right)$ das variações na RPLm e apresentou colinearidade fraca (Quadro 6).

A Ds foi a variável mais importante e também a única que apresentou coeficiente de trilha com valor superior ao do efeito da variável residual, confirmando seu efeito direto, com mesmo sinal, sobre a resistência à penetração do Argissolo na profundidade de $0,20 \mathrm{a}$ 0,40 m (Quadro 6). A AEA apresentou coeficiente do efeito indireto, via Ds, superior ao efeito da variável residual e com sinal negativo, indicando que o seu aumento implica diminuição da resistência do solo à penetração, reforçando a importância dessa variável na avaliação da condição estrutural do solo.

Por serem duas variáveis dependentes da umidade do solo, há relação positiva e linear entre pressão de pré-compactação e resistência do solo à penetração
(Lima et al., 2006). Isso explica os resultados similares da dependência dessas variáveis em relação à qualidade estrutural do solo. Machado et al. (2008) verificaram que a intensificação do uso resultou no aumento da densidade do solo e que essas modificações explicaram mudanças no comportamento da curva característica de retenção de água.

Segundo Lebert \& Horn (1991), a densidade afeta o ângulo de atrito interno por estar relacionada ao arranjamento espacial das partículas, ou seja, o número de pontos de contato entre elas; para um mesmo material, o atrito é tanto maior quanto maior for a densidade. A água também afeta o ângulo de atrito interno, por constituir-se em um agente lubrificante das partículas sólidas do solo. A adição de água ao solo resulta na formação de filmes de moléculas de água ao redor das partículas minerais, reduzindo o atrito entre estas (Lebert \& Horn, 1991).

Portanto, a modificação na distribuição da porosidade do solo pode se somar ao efeito do arranjo espacial das partículas sólidas umedecidas, causando grandes modificações no atrito entre as partículas e,

Quadro 5. Desdobramento das correlações lineares em efeitos diretos e indiretos de atributos físicos e teor de matéria orgânica sobre a pressão de pré-compactação média $\left(\sigma_{\mathrm{p}} \mathrm{m}\right)$ para a profundidade de 0,20 a $0,40 \mathrm{~m}$

\begin{tabular}{|c|c|c|c|c|c|c|c|}
\hline Variável & $\mathrm{S}+\mathrm{A}$ & MOT & PMOP & AGR & DMP & Ds & Total \\
\hline $\mathrm{S}+\mathrm{A}$ & 0,362 & $-0,007$ & 0,003 & $-0,007$ & 0,101 & 0,085 & $0,724 * *$ \\
\hline MOT & 0,082 & $-0,036$ & $-0,004$ & 0,029 & $-0,085$ & $-0,165$ & $-0,115$ \\
\hline PMOP & $-0,095$ & $-0,011$ & $-0,013$ & 0,022 & $-0,120$ & $-0,179$ & $-0,466^{*}$ \\
\hline AGR & $-0,046$ & $-0,020$ & $-0,006$ & 0,051 & $-0,184$ & $-0,333$ & $-0,515^{* *}$ \\
\hline DMP & $-0,172$ & $-0,015$ & $-0,007$ & 0,044 & $-0,212$ & $-0,356$ & $-0,785^{* *}$ \\
\hline Ds & 0,070 & 0,014 & 0,005 & $-0,039$ & 0,172 & 0,440 & $0,688^{* *}$ \\
\hline \multicolumn{4}{|c|}{$\begin{array}{l}\text { Coeficiente de Determinação }\left(\mathrm{R}^{2}\right) \\
\text { Efeito da Variável Residual }(\rho \varepsilon)\end{array}$} & & & & $\begin{array}{l}0,872 \\
0,358\end{array}$ \\
\hline \multicolumn{4}{|c|}{ Colinearidade } & & & & Fraca \\
\hline
\end{tabular}

* correlação de Pearson significativa a $5 \%$, para o efeito total; ** correlação de Pearson significativa a 1 \%, para o efeito total; Leitura dos efeitos diretos (coeficiente de trilha) sobre a variável resposta na diagonal, em negrito, e dos efeitos indiretos na horizontal; - S+A: silte mais argila; MOT: teor de matéria orgânica total; PMOP: porcentagem de matéria orgânica particulada; AGR: porcentagem de agregação em água; DMP: diâmetro médio ponderado de agregados estáveis em água e Ds: densidade do solo.

Quadro 6. Desdobramento das correlações lineares em efeitos diretos e indiretos de atributos físicos e teor de matéria orgânica sobre a resistência do solo à penetração em laboratório média (RPLm) para a profundidade de 0,20 a $0,40 \mathrm{~m}$

\begin{tabular}{|c|c|c|c|c|c|c|}
\hline Variável & $\mathrm{S}+\mathrm{A}$ & MOT & AEA & Ds & GC & Total \\
\hline $\mathrm{S}+\mathrm{A}$ & 0,339 & 0,013 & 0,123 & 0,114 & $-0,020$ & $0,569 * *$ \\
\hline MOT & 0,077 & 0,056 & $-0,172$ & $-0,222$ & 0,030 & $-0,231$ \\
\hline AEA & $-0,116$ & 0,026 & $-0,359$ & $-0,395$ & 0,053 & $-0,791^{* *}$ \\
\hline Ds & 0,065 & $-0,021$ & 0,240 & 0,591 & $-0,104$ & $0,771^{* *}$ \\
\hline GC & 0,060 & 0,015 & 0,168 & 0,547 & $-0,113$ & $0,647 * *$ \\
\hline \multicolumn{3}{|c|}{$\begin{array}{l}\text { Coeficiente de Determinação }\left(\mathrm{R}^{2}\right) \\
\text { Efeito da Variável Residual }(\rho \varepsilon)\end{array}$} & & & & $\begin{array}{l}0,847 \\
0,391\end{array}$ \\
\hline \multicolumn{3}{|c|}{ Colinearidade } & & & & Fraca \\
\hline
\end{tabular}

* correlação de Pearson significativa a $5 \%$, para o efeito total; ** correlação de Pearson significativa a $1 \%$, para o efeito total; Leitura dos efeitos diretos (coeficiente de trilha) sobre a variável resposta na diagonal, em negrito, e dos efeitos indiretos na horizontal; - S+A: silte mais argila; MOT: teor de matéria orgânica total; AEA: porcentagem de agregados estáveis em água; Ds: densidade do solo; e GC: grau de compactação. 
consequentemente, na pressão de pré-compactação e resistência do solo à penetração.

No horizonte $\mathrm{AB}$, a variação nos teores de matéria orgânica entre as áreas estudadas foi muito pequena; a mata teve uma leve tendência a apresentar maior teor de MOT e maior PMOP (Quadro 7).

Por se tratar de um horizonte localizado abaixo da profundidade de preparo do solo, a dinâmica da matéria orgânica é menos influenciada pela retirada da vegetação nativa e introdução da cana-de-açúcar. No entanto, pressões aplicadas pelas extremidades dos órgãos ativos dos implementos de preparo do solo e cultivo, sobre as camadas adjacentes, podem causar compactação subsuperficial, aumentando sua densidade e diminuindo o volume total de poros, principalmente, pela deformação da macroporosidade.

As áreas cultivadas apresentaram maior Ds e menor VTP, e a mata apresentou maior estabilidade de agregados, maior volume de MACRO e menor de MICRO em relação a elas (Quadro 7).

Esses resultados comprovam a importância do efeito desses atributos físicos sobre o comportamento mecânico do horizonte $\mathrm{AB}$ do Argissolo estudado, que apresentou maior $\sigma_{\mathrm{p}} \mathrm{m}$ e RPL quando submetido ao cultivo com cana-de-açúcar (Quadro 7). Entretanto, praticamente não se observou diferença entre as áreas submetidas a diferentes tipos e tempo de cultivo, indicando que o sistema de exploração canavieira adotado pela Usina Coruripe não tem causado compactações adicionais na profundidade de 0,2 a $0,4 \mathrm{~m}$, a partir dos primeiros anos de cultivo.

Nos quadros 8 e 9 são apresentados os resultados dos desdobramentos das correlações dos atributos físicos e teor de matéria orgânica com a pressão de pré-compactação média e resistência do solo à penetração média em laboratório, para a profundidade de 0,40 a 0,60 m. O conjunto de variáveis independentes apresentou colinearidade fraca e explicou 74,1 e 58,9 \% do comportamento da $\sigma_{\mathrm{p}} \mathrm{m}$ e RPLm, respectivamente.

A densidade do solo e o grau de compactação apresentaram coeficiente de correlação significativo e positivo com a $\sigma_{\mathrm{p}} \mathrm{m}$ do horizonte Bt (Quadro 8). Não foi observada nenhuma correlação entre atributos referentes à agregação e teores de matéria orgânica do solo com a pressão de pré-compactação e resistência do solo à penetração.

Quadro 8. Desdobramento das correlações lineares em efeitos diretos e indiretos de atributos físicos e teor de matéria orgânica sobre a pressão de précompactação média $\left(\sigma_{\mathrm{p}} \mathrm{m}\right)$ para a profundidade de 0,40 a $0,60 \mathrm{~m}$

\begin{tabular}{lcccccc}
\hline Variável & S+A & MOT & AGR & Ds & GC & Total \\
\hline S+A & $\mathbf{0 , 2 5 9}$ & 0,002 & 0,004 & $-0,074$ & $-0,367$ & $-0,176$ \\
MOT & 0,040 & $\mathbf{0 , 0 0 9}$ & 0,009 & $-0,036$ & $-0,158$ & $-0,136$ \\
AGR & 0,047 & 0,004 & $\mathbf{0 , 0 2 3}$ & $-0,033$ & $-0,063$ & $-0,022$ \\
Ds & $-0,143$ & $-0,002$ & $-0,007$ & $\mathbf{0 , 1 3 4}$ & 0,780 & $0,762^{* *}$ \\
GC & $-0,115$ & $-0,002$ & $-0,002$ & 0,127 & $\mathbf{0 , 8 2 4}$ & $0,832^{* *}$ \\
Coeficiente de Determinação $\left(\mathrm{R}^{2}\right)$ & & & 0,741 \\
Efeito da Variável Residual $(\rho \varepsilon)$ & & & 0,509 \\
\multicolumn{7}{l}{ Colinearidade }
\end{tabular}

* correlação de Pearson significativa a $5 \%$, para o efeito total; ** correlação de Pearson significativa a $1 \%$, para o efeito total; - Leitura dos efeitos diretos (coeficiente de trilha) sobre a variável resposta na diagonal, em negrito, e dos efeitos indiretos na horizontal; - S+A: silte mais argila; MOT: teor de matéria orgânica total; AGR: porcentagem de agregação em água; Ds: densidade do solo; e GC: grau de compactação.

Quadro 7. Pressão de pré-compactação média, resistência do solo à penetração em laboratório média, atributos físicos e teor de matéria orgânica do Argissolo dos seis tratamentos na profundidade de 0,20 a $0,40 \mathrm{~m}$

\begin{tabular}{|c|c|c|c|c|c|c|}
\hline \multirow{2}{*}{ Variável } & \multicolumn{6}{|c|}{ Tratamento } \\
\hline & Mata & $4 \operatorname{anos} I$ & $14 \operatorname{anos} I$ & $14 \operatorname{anos} V$ & $30 \operatorname{anos} I$ & 30 anos $V$ \\
\hline$\sigma_{\mathrm{p}} \mathrm{m}(\mathrm{kPa})$ & 50 & 99 & 82 & 77 & 88 & 88 \\
\hline RPLm (kPa) & 1114 & 2289 & 2412 & 2178 & 2215 & 2423 \\
\hline $\mathrm{S}+\mathrm{A}\left(\mathrm{g} \mathrm{kg}^{-1}\right)$ & 207 & 299 & 250 & 232 & 253 & 276 \\
\hline $\operatorname{MOT}\left(\mathrm{g} \mathrm{kg}^{-1}\right)$ & 8,74 & 8,33 & 8,28 & 7,73 & 7,46 & 7,45 \\
\hline PMOP (\%) & 7,51 & 5,03 & 5,79 & 5,71 & 4,42 & 3,66 \\
\hline PMOC (\%) & 92,49 & 94,97 & 94,21 & 94,29 & 95,58 & 96,34 \\
\hline AGR (\%) & 66,41 & 43,90 & 34,23 & 32,05 & 36,98 & 29,90 \\
\hline AEA (\%) & 85,20 & 70,84 & 65,38 & 69,76 & 68,23 & 60,01 \\
\hline $\mathrm{DMP}(\mathrm{mm})$ & 1,73 & 0,67 & 0,59 & 0,78 & 0,68 & 0,53 \\
\hline $\operatorname{VTP}\left(\mathrm{m}^{3} \mathrm{~m}^{-3}\right)$ & 0,45 & 0,39 & 0,39 & 0,39 & 0,40 & 0,41 \\
\hline MICRO (\%) & 37,76 & 64,41 & 54,17 & 51,66 & 53,90 & 53,48 \\
\hline MACRO (\%) & 62,24 & 35,59 & 45,83 & 48,34 & 46,10 & 46,52 \\
\hline Ds $\left(\mathrm{kg} \mathrm{dm}^{-3}\right)$ & 1,41 & 1,58 & 1,58 & 1,58 & 1,55 & 1,53 \\
\hline GC $(\%)$ & 73,49 & 81,17 & 79,49 & 81,35 & 78,21 & 78,32 \\
\hline
\end{tabular}

$\sigma_{\mathrm{p}} \mathrm{m}$ : pressão de pré-compactação média; RPLm: resistência do solo à penetração em laboratório média; S+A: silte mais argila; MOT: matéria orgânica total; PMOP: porcentagem de matéria orgânica particulada; PMOC: porcentagem de matéria orgânica complexada; AGR: porcentagem de agregação em água; AEA: porcentagem de agregados estáveis em água; DMP: diâmetro médio ponderado de agregados estáveis em água; VTP: volume total de poros; MICRO: porcentagem microporos; MACRO: porcentagem macroporos; Ds: densidade do solo; e GC: grau de compactação. 
O GC foi a variável mais importante na relação causa e efeito com a $\sigma_{\mathrm{p}} \mathrm{m}$, apresentando coeficiente de trilha maior do que o efeito da variável resíduo e com mesmo sinal e magnitude muito similar à do coeficiente do efeito total (Quadro 8). A Ds não apresentou importância no efeito direto sobre a $\sigma_{\mathrm{p}} \mathrm{m}$, porém mostrou grande efeito indireto via grau de compactação, indicando também a importância dessa variável explicativa sobre as alterações na $\sigma_{\mathrm{p}} \mathrm{m}$.

Nenhuma das variáveis explicativas selecionadas para a análise de trilha apresentou coeficiente de correlação significativo com a RPLm, ou seja, todas as variáveis independentes mostraram efeito total menor do que o efeito da variável residual (Quadro 9).No entanto, a Ds apresentou efeito direto e o GC efeito indireto, via Ds, maiores do que o efeito da variável residual, indicando a importância da correlação de causa e efeito dessas duas variáveis com a RPLm do horizonte Bt.

No quadro 10, pode-se observar pequeno aumento na $\sigma_{\mathrm{p}} \mathrm{m}$ e na RPLm no horizonte Bt do solo das áreas cultivadas em relação à mata; as áreas cultivadas não apresentaram variação lógica entre elas, que poderia relacionar a compactação dessa camada com o tempo ou tipo de cultivo. Contudo, pode-se inferir que o sistema de cultivo com cana-de-açúcar teve pequeno efeito sobre a compactação na profundidade de 0,40 a 0,60 m.

Quadro 9. Desdobramento das correlações lineares em efeitos diretos e indiretos de atributos físicos e teor de matéria orgânica sobre a resistência do solo à penetração média em laboratório (RPLm) para a profundidade de 0,40 a $0,60 \mathrm{~m}$

\begin{tabular}{lccccccc}
\hline Variáveis & S+A & MOT & PMOP & AGR & Ds & GC & Total \\
\hline S+A & $\mathbf{0 , 4 8 6}$ & $-0,065$ & 0,001 & 0,014 & $-0,438$ & 0,043 & 0,041 \\
MOT & 0,075 & $\mathbf{- 0 , 4 2 0}$ & $-0,002$ & 0,033 & $-0,213$ & 0,019 & $-0,508$ \\
PMOP & $-0,077$ & $-0,125$ & $\mathbf{- 0 , 0 0 6}$ & 0,018 & $-0,113$ & 0,019 & $-0,284$ \\
AGR & 0,089 & $-0,186$ & $-0,001$ & $\mathbf{0 , 0 7 4}$ & $-0,194$ & 0,007 & $-0,211$ \\
Ds & $-0,268$ & 0,112 & 0,001 & $-0,018$ & $\mathbf{0 , 7 9 3}$ & $-0,091$ & 0,529 \\
GC0 & $-0,216$ & 0,081 & 0,001 & $-0,006$ & 0,750 & $\mathbf{- 0 , 0 9 7}$ & 0,513 \\
Coeficiente de Determinação $\left(R^{2}\right)$ & & & & & & 0,589 \\
Efeito da Variável Residual $(\rho \varepsilon)$ & & & & & & 0,641 \\
Colinearidade & & & & & & Fraca \\
\hline
\end{tabular}

* correlação de Pearson significativa a $5 \%$, para o efeito total; ** correlação de Pearson significativa a $1 \%$, para o efeito total; Leitura dos efeitos diretos (coeficiente de trilha) sobre a variável resposta na diagonal, em negrito, e dos efeitos indiretos na horizontal; - S+A: silte mais argila; MOT: teor de matéria orgânica total; PMOP: porcentagem de matéria orgânica particulada; AGR: porcentagem de agregação em água; Ds: densidade do solo; e GC: grau de compactação.

Quadro 10. Pressão de pré-compactação média, resistência do solo à penetração em laboratório média, atributos físicos e teor de matéria orgânica do Argissolo dos seis tratamentos na profundidade de 0,40 a $0,60 \mathrm{~m}$

\begin{tabular}{|c|c|c|c|c|c|c|}
\hline \multirow{2}{*}{ Variável } & \multicolumn{6}{|c|}{ Tratamento } \\
\hline & Mata & $4 \operatorname{anos} I$ & $14 \operatorname{anos} I$ & $14 \operatorname{anos} V$ & $30 \operatorname{anos} I$ & $30 \operatorname{anos} V$ \\
\hline$\sigma_{\mathrm{p}} \mathrm{m}(\mathrm{kPa})$ & 86 & 131 & 85 & 113 & 131 & 103 \\
\hline RPLm (kPa) & 1991 & 2598 & 2509 & 2243 & 2249 & 2261 \\
\hline $\mathrm{S}+\mathrm{A}\left(\mathrm{g} \mathrm{kg}^{-1}\right)$ & 369 & 404 & 408 & 394 & 392 & 407 \\
\hline $\operatorname{MOT}\left(\mathrm{g} \mathrm{kg}^{-1}\right)$ & 8,12 & 7,62 & 6,49 & 7,84 & 7,06 & 7,21 \\
\hline PMOP (\%) & 8,54 & 5,37 & 5,60 & 6,26 & 5,72 & 5,99 \\
\hline PMOC (\%) & 91,46 & 94,63 & 94,40 & 93,74 & 94,28 & 94,01 \\
\hline AGR (\%) & 68,33 & 62,75 & 54,79 & 65,03 & 59,96 & 59,12 \\
\hline AEA (\%) & 84,70 & 74,08 & 74,38 & 84,72 & 79,80 & 78,12 \\
\hline $\mathrm{DMP}(\mathrm{mm})$ & 1,41 & 1,21 & 0,77 & 1,25 & 1,01 & 0,89 \\
\hline $\operatorname{VTP}\left(\mathrm{m}^{3} \mathrm{~m}^{-3}\right)$ & 0,46 & 0,43 & 0,46 & 0,43 & 0,43 & 0,45 \\
\hline MICRO (\%) & 52,08 & 68,10 & 56,12 & 63,25 & 63,89 & 59,17 \\
\hline MACRO (\%) & 47,92 & 31,90 & 43,88 & 36,75 & 36,11 & 40,83 \\
\hline Ds $\left(\mathrm{kg} \mathrm{dm}^{-3}\right)$ & 1,40 & 1,46 & 1,39 & 1,46 & 1,48 & 1,41 \\
\hline GC (\%) & 72,98 & 77,78 & 73,19 & 78,10 & 77,95 & 75,03 \\
\hline
\end{tabular}

$\sigma_{\mathrm{p}} \mathrm{m}$ : pressão de pré-compactação média; RPLm: resistência do solo à penetração em laboratório média; S+A: silte mais argila; MOT: matéria orgânica total; PMOP: porcentagem de matéria orgânica particulada; PMOC: porcentagem de matéria orgânica complexada; AGR: porcentagem de agregação em água; AEA: porcentagem de agregados estáveis em água; DMP: diâmetro médio ponderado de agregados estáveis em água; VTP: volume total de poros; MICRO: porcentagem microporos; MACRO: porcentagem macroporos; Ds: densidade do solo; e GC: grau de compactação. 


\section{CONCLUSÕES}

1. Nas três profundidades estudadas, o grau de compactação e a densidade do solo são os atributos que apresentam maior relação de causa e efeito com a pressão de pré-compactação e resistência à penetração do Argissolo Amarelo Distrocoeso estudado.

2. O grau de compactação foi a variável que apresentou maior relação causa e efeito direto sobre a pressão de pré-compactação e resistência a penetração na profundidade de 0 a 0,20 m do Argissolo Amarelo.

3. O cultivo com cana-de-açúcar diminuiu o teor de matéria orgânica e estabilidade de agregados em água do horizonte superficial do Argissolo Amarelo, o que aumentou a pressão de pré-compactação e a resistência do solo à penetração, principalmente por meio do aumento da densidade do solo e do grau de compactação.

4. O aumento na densidade do solo e no grau de compactação mostrou relação causa e efeito direto com o aumento da pressão de pré-compactação e resistência à penetração dos horizontes $\mathrm{AB}$ e $\mathrm{Bt}$ do Argissolo Amarelo cultivado com cana-de-açúcar.

5. A matéria orgânica e a estabilidade de agregados tiveram pouca ou nenhuma importância na causa e efeito sobre a pressão de pré-compactação e resistência à penetração dos horizontes subsuperficiais do Argissolo Amarelo estudado.

\section{AGRADECIMENTOS}

À Usina Coruripe, pela disponibilização das áreas e pelo apoio logístico.

\section{LITERATURA CITADA}

ASSOCIAÇÃO BRASILEIRA DE NORMAS TÉCNICA - ABNT. Solo: Ensaio de compactação - NBR 7182. Rio de Janeiro, 1986. 10p.

ASSOCIAÇÃO BRASILEIRA DE NORMAS TÉCNICA - ABNT. Ensaio de adensamento unidimensional. ABNT. - NBR 12007. Rio de Janeiro, 1990. 13p.

BIGNER, R.L. \& WELLS, L.G. Compact-a reclamation soil compaction model. Part I, Model development. Trans. Am. Soc. Agron. Eng, 53:405-413, 1992.

BRAIDA, J.A.; REICHERT, J.M.; VEIGA, M. \& REINERT, D.J. Resíduos vegetais na superfície e carbono orgânico do solo e suas relações com a densidade máxima obtida no ensaio de proctor. R. Bras. Ci. Solo, 30:605-614, 2006.

CAMBARDELLA, C.A. \& ELLIOT, E.T. Particulate soil organic matter changes across a grassland cultivation sequence. Soil Sci. Soc. Am. J., 56:777-783, 1992.

CAPUTO, H.P. Mecânica dos solos e suas aplicações. 4.ed. Rio de Janeiro, Livros Técnicos e Científicos, 1980. v.1. 219p.
CRUZ, C.D.; REGAZZI, A.J. \& CARNEIRO, P.C.S. Modelos biométricos aplicados ao melhoramento genético. 3.ed. Viçosa, MG, Universidade Federal de Viçosa, 2004. 480p.

D'AGOSTINI, L.R. Variações temporais da coesividade entre agregados de solo. Porto Alegre, Universidade Federal do Rio Grande do Sul, 1992. 82p. (Tese de Doutorado)

DIAS JUNIOR, M.S. Compression of three soils under longterm tillage and wheel traffic. East Lansing, Michigan State University, 1994. 114p. (Tese de Doutorado)

DIAS JUNIOR, M.S. Compactação do solo. In: NOVAIS, R.F.; ALVAREZ V., V.H. \& SHAEFER, C.E.G.R. Tópicos em ciência do solo. Viçosa, MG, Sociedade Brasileira de Ciência do Solo, 2000. v.1.p.56-94.

DIAS JUNIOR, M.S. \& PIERCE, F.J. Revisão de literatura: O processo de compactação do solo e sua modelagem. R. Bras. Ci. Solo, 20:175-182, 1996.

EMPRESA BRASILEIRA DE PESQUISA AGROPECUÁRIA EMBRAPA. Centro Nacional de Pesquisa dos Solos. Manual de métodos de análise de solo. 2.ed. Rio de Janeiro, 1997. 212p.

EMPRESA BRASILEIRA DE PESQUISA AGROPECUÁRIA EMBRAPA. Centro Nacional de Pesquisa dos Solos. Manual de análises químicas de solos, plantas e fertilizantes. Brasília, 1999. 370p.

EMPRESA BRASILEIRA DE PESQUISA AGROPECUÁRIA EMBRAPA. Centro Nacional de Pesquisa do Solo. Sistema brasileiro de classificação de solos. 2.ed. Rio de Janeiro, 2006. 300p.

ETANA, A.; COMIA, R.A. \& HAKANSSON, I. Effects of uniaxial stress on the physical properties of four Swedish soils. Soil Tillage Res., 44:13-21, 1997.

GOMES, T.C.A. Análise de trilha no estudo de fatores físicos e químicos relacionados ao adensamento e, ou, à compactação em dois solos do norte de Minas Gerais. Viçosa, MG, Universidade Federal de Viçosa, 1996. 105p. (Tese de Mestrado)

GUPTA, S.C. \& ALLMARAS, R.R. Models to access the susceptibility soil to excessive compaction, Adv. Soil Sci., 6:65-100, 1987

HORN, R. \& LEBERT. M. Soil compactability and compressibility. In: SOANE, B.D. \& van OUWERKERK, C., eds. Soil compaction in crop production, London, Elsevier, 1994. p.45-69 (Desevelopments in Agricultural Engineering, 11)

HORN, R.; TAUBNER, H.; WUTTKE, M. \& BAUMGARTL, T. Soil physical properties related to soil structure. Soil Tillage Res., 30:187-216,1993.

JACOMINI, P.K.T.; CAVALCANTI, A.C.; PESSOA, S.C.P. \& SILVEIRA, C.O. Levantamento exploratório. reconhecimento de solos do estado de Alagoas. Recife, Embrapa, Centro de Pesquisas Pedológicas, 1975. 531p. (Boletim Técnico, 35)

KEMPER, W.D. \& ROSENAU, R.C. Aggregate stability and size distribution. In: KLUTE, A., ed. Methods of soil analysis. Madison, American Society of Agronomy, 1986. Part 1. p.425-442. (Agronomy, 9) 
LEBERT, M. \& HORN, R. A method to predict the mechanical strenght of agricultural soils. Soil Tillage Res., 19:275286, 1991.

LIMA, C.L.R. \& SILVA, A.P. Estimativa da capacidade de suporte de carga do solo a partir da avaliação da resistência à penetração. R. Bras. Ci. Solo, 30-2:217-223, 2006.

LIMA, C.L.R.; SILVA, A.P.; IMHOFF, S.; LIMA, H.V. \& LEÃO, T.P. Estimativa da capacidade de suporte de carga do solo a partir da avaliação da resistência à penetração. R. Bras. Ci. Solo, 30:217-223, 2006.

MACHADO, J.L.; TORMENA, A.C.; FIDALSKI, J. \& SCAPIM, C.A. Inter-relações entre propriedades físicas e os coeficientes da curva de retenção de água de um Latossolo sob diferentes sistemas de uso. R. Bras. Ci. Solo, 32:495$502,2008$.
PACHECO, E.P. Estudo da compressibilidade e qualidade de um Argissolo Amarelo cultivado com cana-de-açúcar nos Tabuleiros Costeiros de Alagoas. Recife, Universidade Federal Rural de Pernambuco, 2010. 106p. (Tese de Doutorado)

REINERT, D.J.; ROBAINA, A. \& REICHERT, J.M. Compress - software e proposta de modelo para descrever a compressibilidade dos solos e seus parâmetros. In: CONGRESSO BRASILEIRO DE CIÊNCIA DO SOLO, 29., Ribeirão Preto, 2003. Anais... Ribeirão Preto, Sociedade Brasileira de Ciência do Solo, 2003. CD-ROM.

RÖMKENS, M.J.M. \& MILLER, R.D. Predicting root size and frequency from one-dimensional consolidation data - A mathematical model. Plant Soil, 35:237-248, 1971.

ZHANG, H.; HARTGE, K.H. \& RINGE, H. Effectiveness of organic matter incorporation in reducing soil compactability. Soil Sci. Soc. Am. J., 61:239-245, 1997. 